\title{
Nuclear Magnetic Resonance Evaluation Study for Effect of Foam flooding in Heterogeneous Cores
}

\author{
Shuai Hua ${ }^{1,2}$, Yuan $\mathrm{Li}^{1 *}$, Qinfeng $\mathrm{Di}^{1}$ \\ ${ }^{1}$ School of Mechanics and Engineering Science, Shanghai University,Shanghai,200072,China. \\ ${ }^{2}$ Suzhou Testniumag Corporation Co. Ltd., Suzhou, Jiangsu Province, 215000, China
}

\begin{abstract}
Foam flooding demonstrated the ability to solve the viscous fingering problem of gas flooding and increase the sweep efficiency in enhancing oil recovery. It is commonly used in development of heterogeneous reservoirs. While the characteristics of fluid migration in pores and between layers were still unclear. In this paper, Dynamic change of oil and water with different foam quality was tested during foam flooding by NMR method. Oil displacement effect of water flooding and foam flooding was compared. The results showed the foam quality affected the foam stability and profile control effect. Compared with water flooding, the foam could increase the recovery rate of the low-permeability layer, and the foam system with high stability had a high sweep efficiency and a high oil displacement efficiency in the heterogeneous cores.
\end{abstract}

\section{Introduction}

Reservoirs exhibit different heterogeneities due to differences in reservoir porosity and permeability ${ }^{[1]}$. In development of heterogeneous reservoirs, gravity override and viscous fingering of gas flooding is prominent, which seriously affects oil displacement efficiency. The foam has the characteristics of low density and high apparent viscosity, it is elongated and changed shape through the throat with a small radius ${ }^{[2]}$. This deformation will consume a portion of the energy, which slows the bubble motion and adds extra resistance. Thus, the viscosity of the foam is several orders of magnitude greater than the viscosity of the gas. Many scholars have concluded that the foam first enters and blocks the high-permeability layer during the flow, and then enters the low-permeability layer to improve the oil displacement effect $^{[3-5]}$. Therefore, foam flooding is widely used in the development of heterogeneous reservoirs.

Traditional displacement methods cannot reflect the fluid flow characteristics inside the core. NMR is a novel method to study the flow of different fluids in porous media. It can be commonly used in the experimental study of pore throat structure, size distribution, and fluid flow in rock media with similar reservoir conditions. In this paper, Low-field nuclear magnetic resonance equipment was used to test the changes in the oil and water content of different pores and different layers of heterogeneous cores during the foam injection process. The experimental results were used to evaluate the displacement and profile control effects of the foam systems.

\section{Theoretical basis}

\subsection{Theory of NMR}

Combining nuclear magnetic resonance technology with traditional foam displacement experimental methods, a foam flooding NMR experimental can be conducted. Nuclear magnetic resonance is a method to calculate the amount of hydrogen nuclei and the volume of fluid by measuring the fluid magnetization decay with time ( the change in relaxation time $T_{2}$ ) in an external magnetic field ${ }^{[6]}$. The NMR technique obtains the nuclear magnetic resonance $T_{2}$ spectrum by measuring the superposition of the signals of water in different sizes of pores. $T_{2}$ value corresponding to the peak of the relaxation curve is proportional to the pore size of the liquid in the core, and the enclosed peak area is proportional to the liquid mass in the core ${ }^{[7]}$.

\subsection{Classification of NMR signals between oil and water}

Due to overlap of the nuclear magnetic resonance relaxation time of oil and water in cores, the nuclear magnetic signals of water phase and oil phase cannot be clearly distinguished in $\mathrm{T}_{2}$ spectrum. When $\mathrm{MnCl}_{2}$ aqueous solution was used instead of water for displacement, the direct contact between $\mathrm{Mn}^{2+}$ and $\mathrm{H}$ protons in water would cause spin exchange, which could accelerate the relaxation of $\mathrm{H}$ protons in water. The best effect of distinguishing oil and water $\mathrm{T}_{2}$ spectrum and images can be obtained by using a $0.5 \mathrm{wt} \% \mathrm{MnCl}_{2}$

*Corresponding author's e-mail: 2017rhythm@shu.edu.cn 
aqueous solution instead of pure water for flooding in our test ${ }^{[8]}$. When the foam is injected, no nuclear magnetic signal was detected for gas phase in the foam by adding $\mathrm{MnCl}_{2}$ to the foaming agent. Nuclear magnetic signals between foam liquid (surfactant solution) and oil phase can be distinguished.

\section{Experimental devices experimental method}

The experimental device mainly includes: a nitrogen bottle, a gas flow massmeter, a constant flow pump, a nuclear magnetic displacement device unit, an intermediate container, and a control system(as figure1). The experimental materials include surfactant (CTAB), manganese chloride $\left(\mathrm{MnCl}_{2}\right)$, diesel, heavy water $\left(\mathrm{D}_{2} \mathrm{O}\right)$. 3 heterogeneous artificial cores(core $01 \sim$ core 03 )were made by pressing two kinds of quartz sand of $10 \mu \mathrm{m} \sim$ $20 \mu \mathrm{m}$ and $120 \mu \mathrm{m} \sim 200 \mu \mathrm{m}$. As shown in the figure 2, the heterogeneous core consists of two parts, high-permeability layer and low-permeability layer. It is evenly distributed along the sagittal plane and the cross section, with a diameter of $2.5 \mathrm{~cm}$ and a length of about 9 $\mathrm{cm}$.

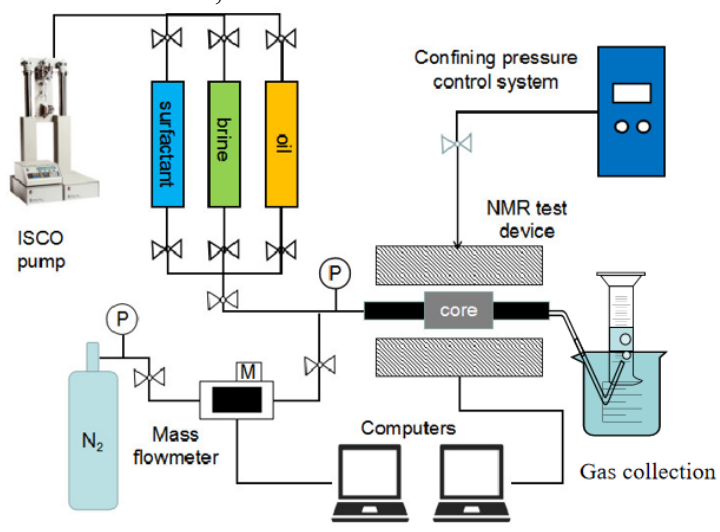

Figure 1. Experimental apparatus for foam dynamic stability evaluation
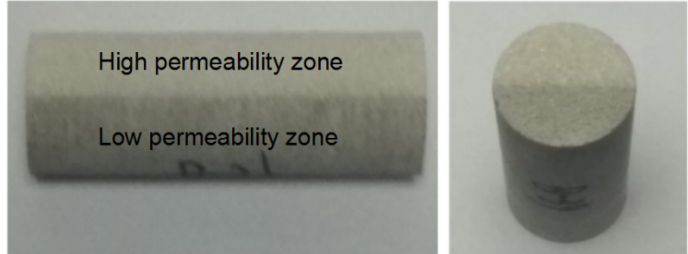

Figure 2. Schematic diagram of the sagittal plane and cross section of the heterogeneous core

The experimental procedure is as follows:

1) Heterogeneous sand cores were put into a constant temperature drying oven and vacuumed for 12 hours.

2) The cores were saturated with diesel oil after they were dried.

3) In order to distinguish the surfactant solution of the foam and the original water content before foam flooding, the core was flooded by heavy water(contain $0.5 \mathrm{wt} \%$ $\left.\mathrm{MnCl}_{2}\right), \mathrm{T}_{2}$ spectrum test were tested during the flooding process.

4) foaming agent (containing $0.5 \%$ CTAB surfactant and $0.5 \% \mathrm{MnCl}_{2}$ ) was used to prepare foam. The total flow rate of gas and liquid was $1 \mathrm{~mL} / \mathrm{min}$. After the flow rate is stabilized, 10.0 PV of gas and water was injected into the core, the outlet gas volume, $\mathrm{T}_{2}$ spectrum were measured and tested during foam injection process. The gas volume content in the core is calculated according to the cumulative volume of the inlet gas flow meter and the recorded volume of the outlet. The oil saturation of different layers is measured according to the sequence SE-SPI.

\section{Results and discussion}

\subsection{Characteristics of water flooding}

$15 \mathrm{PV}$ of heavy water was injected at a constant flow rate of $1 \mathrm{~mL} / \mathrm{min}$, and the volume of diesel at the outlet and the $T_{2}$ spectrum of cores at different times were recorded in Figure3. 


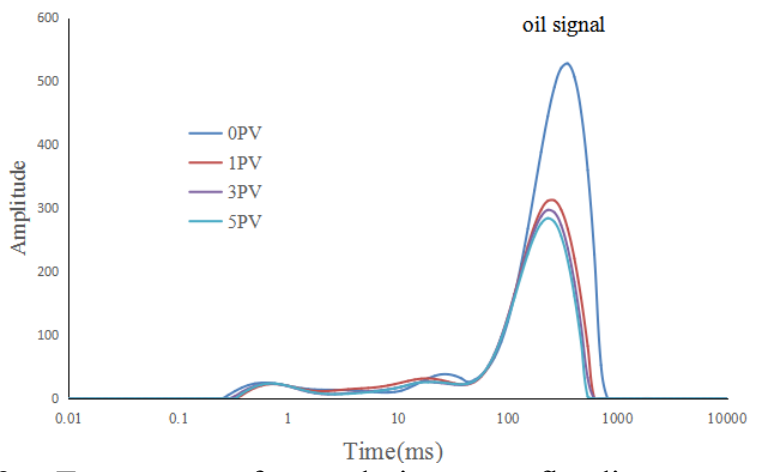

Figure 3. $T_{2}$ spectrum of cores during water flooding process(core 01 )

Since there is no nuclear magnetic resonance signal detected in heavy water, the $T_{2}$ spectrum only demonstrates the change of oil in the water flooding process. It can be seen from Figure 6 that the oil signal is mainly distributed in the range of $100 \mathrm{~ms}-1000 \mathrm{~ms}$. During 1.0PV of water injection, the oil peaks in graph a and graph $b$ decreased rapidly, and the oil peaks during the water flooding 1.0PV 5.0PV showed less variation, indicating that the oil displacement efficiency in the core after the water flooding 1.0PV was much smaller. During the water flooding process, the oil peaks were gradually shifted to the left. Because the oil in the large pores was driven out of the core, and the proportion of oil in the small pores was gradually increased. That caused the peak of oil signal shifted to the small relaxation time .
As shown in the figure4, there are two peaks in the $T_{2}$ spectrum during the foam flooding process. The peak on the left (low $\mathrm{T}_{2}$ value) is signal of $\mathrm{MnCl}_{2}$ solution(water signal), and the oil signal is on the right. The water peaks of core 01 and core 02 increase rapidly After 2.0PV foam injection and almost unchanged when foam injection continued, while the water peaks of core 03 remained increased with time. For a foam system with foam quality of $90 \%$, because the rate of foam coalescence increase [9], the plugging effect and the ability of gas to occupy the pores is will be weak than other foam systems. The oil peaks of three cores mainly drops during $0.0 \mathrm{PV}$ to $5.0 \mathrm{PV}$, and shift to larger relaxation time contrary to water flooding. This shows that the foam can mobilize the oil in the small pores to a certain extent.

\subsection{Characteristics of the $T_{2}$ spectrum of foam flooding}

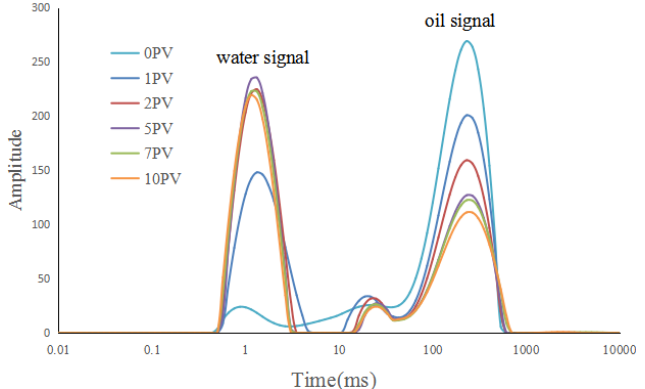

(a) core number: 01 , foam quality of $50 \%$

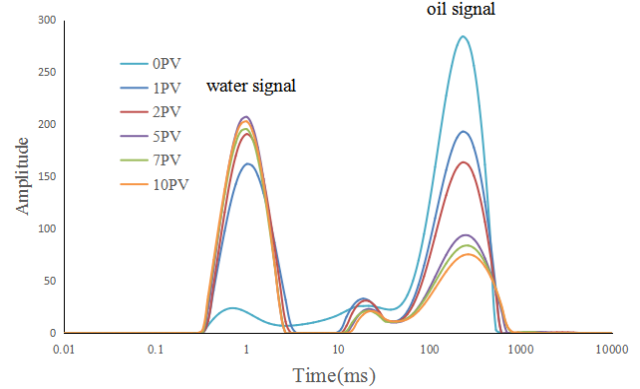

(b) core number:02, foam quality of $66.7 \%$

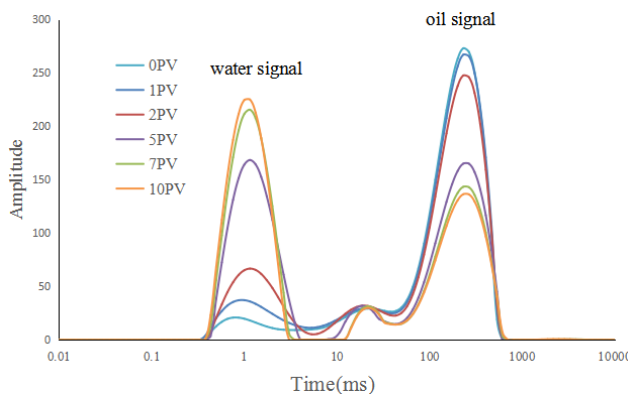

(a) core number: 03 , foam quality of $90 \%$

Figure 4. $T_{2}$ spectrum of cores during water flooding process displacement efficiency after water flooding. The foam flooding had a higher oil displacement efficiency during $5 \mathrm{PV}-10 \mathrm{PV}$, and the oil recovery curve tended to be gentle at late stage. It can be seen the oil displacement efficiency of foam system with foam quality of $90 \%$ was

The results in Figure5 shows that the foam flooding carried out by the three cores had a certain oil 
significantly higher than that with foam quality of $90 \%$, they also have the higher oil recovery rate in the low permeability layer after foam flooding(table1). It illustrated the better performance of foam system in profile control effect ${ }^{[10-11]} . \Delta V_{g}$ in the table 1 shows the volumetric content of gas in the core after foam injection. It indicates that the foam system with foam quality of $90 \%$ has the weakest dynamic stability due to lowest accumulated gas saturation in the core. The defoaming effect of residual oil maybe more significant ${ }^{[12]}$.

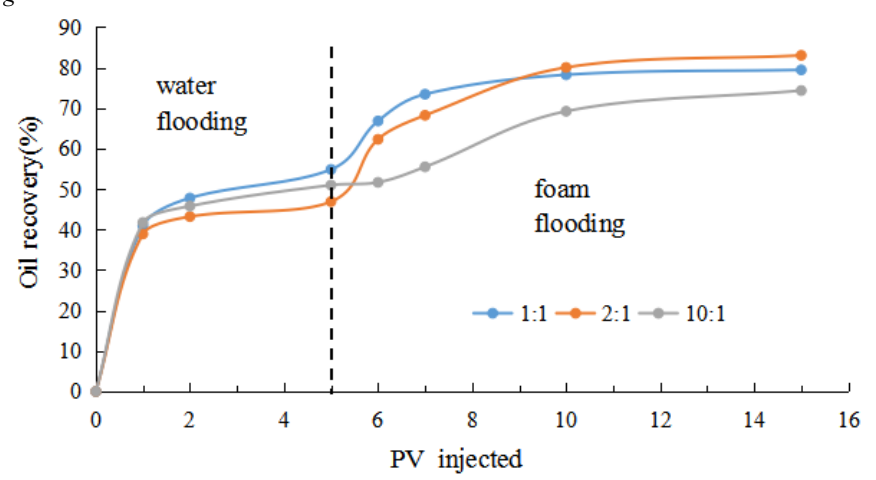

Figure5 Oil recovery according to $T_{2}$ spectrum

Table1 Experimental results after foam flooding

\begin{tabular}{|c|c|c|c|}
\hline $\begin{array}{c}\text { Core number/ } \\
\text { Foam quality }\end{array}$ & $\begin{array}{c}\text { Oil recovery of } \\
\text { high-permeability layer } \\
(\% \text { OIP })\end{array}$ & $\begin{array}{c}\text { Oil recovery of } \\
\text { low-permeability layer } \\
(\% \text { OOIP })\end{array}$ & $\begin{array}{c}\Delta V_{g} \\
(\mathrm{~mL})\end{array}$ \\
\hline $01 / 50 \%$ & 54 & 92 & 3.2 \\
\hline $02 / 66.7 \%$ & 36 & 87 & 4.6 \\
\hline $03 / 90 \%$ & 69 & 84 & 1.8 \\
\hline
\end{tabular}

\section{Conclusion}

The foam could increase the recovery rate of the low small pores and permeability layer of the heterogeneous core compared with the water flooding and had a certain profile control effect. Foam quality has a great influence on the dynamic stability of foam core and profile control efficiency in the heterogeneous core. The foam with higher foam quality could produce stronger foam and continuous oil displacement effect. However, when the gas and liquid were relatively large, the dynamic stability of the foam was greatly reduced due to the coalescence of foam.

\section{Acknowledgments}

The authors would like to thank the National Natural Science Foundation of China (Youth Fund) $(51804193,51704190)$ for the financial support.

\section{References}

1. Elizabeth Mas Hernández, P. Grassia , and N. Shokri . "Modelling foam improved oil recovery within a heterogeneous reservoir." Colloids \& Surfaces A Physicochemical \& Engineering Aspects 510(2016):43-52.

2. Jones, S. A. , et al. "Effect Of Surfactant Concentration On Foam: From Coreflood Experiments To Implicit-Texture Foam-Model Parameters." Journal of Industrial \& Engineering Chemistry 37(2016):268-276.

3. Guo, Feng, and S. A. Aryana . "Improved sweep efficiency due to foam flooding in a heterogeneous microfluidic device." Journal of Petroleum Science and Engineering 164(2018):155-163.

4. Yekeen N, Manan M A, Idris A K, et al. A comprehensive review of experimental studies of nanoparticles-stabilized foam for enhanced oil recovery $[\mathrm{J}]$. Journal of Petroleum Science and Engineering, 2018, 164:43-74.

5. Kovscek A R, Bertin H J . Foam Mobility in Heterogeneous Porous Media[J]. Transport in Porous Media, 2003, 52(1):17-35.

6. Xue D J, Zhou H W, Liu Y T, et al. Study of Drainage and Percolation of Nitrogen - Water Flooding in Tight Coal by NMR Imaging[J]. Rock Mechanics and Rock Engineering, 2018, 51(11): $3421-3437$.

7. Di, Qinfeng, et al. "Visualization experiments on polymer-weak gel profile control and displacement by NMR technique." Petroleum Exploration and Development 44.2(2017):294-298.

8. Jingnan ZHANG, et al, Nuclear magnetic resonance experiments on foam flooding and evaluation of foam dynamic stability, Petroleum Exploration and Development,2018,45(5): 910-917.

9. Sheng J J, Herd B L, Sheng J J, et al. Critical review of field EOR projects in shale and tight reservoirs $[\mathrm{J}]$. Journal of Petroleum Science \& Engineering, $2017, \quad 159$

10. Siddiqui, S. , Talabani, S. , Yang, J. , Saleh, S. T. , \& Islam, M. R. . (2003). An experimental investigation of the diversion characteristics of foam in berea sandstone cores of contrasting permeabilities. 
Journal of Petroleum Science and Engineering, 37(s $1-2), 51-67$.

11. Singh R , Mohanty K K . Foam flow in a layered, heterogeneous porous medium: A visualization study[J]. Fuel, 2017, 197:58-69.

12. Osei-Bonsu K, Grassia P, Shokri N . Investigation of foam flow in a 3D printed porous medium in the presence of oil[J]. Journal of Colloid and Interface Science, 2017, 490:850-858. 